\title{
A Study of Hotel Notices and Signs Translation from the Perspective of Text Typology
}

\author{
Fei FANG ${ }^{1}$, Xiaoxiao SHEN ${ }^{2}$ \\ ${ }^{1)}$ College of Foreign Studies, Shandong Institute of Business and Technology, Yantai, Shandong (youyouxiaojun@aliyun.com) \\ ${ }^{2)}$ Department of Foreign Languages, Wenjing College, Yantai University, Yantai, Shandong (shenxiaoxiao666@ 163.com)
}

\begin{abstract}
With the further advancement of economic globalization, there have been increasingly constant contacts between nations in the field of business, travel, culture, politics, etc. As a temporary home of foreign travelers in China, hotels and the bilingual services provided by them are increasingly important. Based on the theory of text typology and text functions, this essay will probe into the text features, functions, translation principles and problems in existence with the translation of hotel notices and public signs, in the hope of offering some advices for the contemporary bilingual environment of hotels in China.
\end{abstract}

Keywords - text typology, text function, intertextuality, translation principles, loan translation

\section{文本类型学视角下的酒店公示语翻译研究

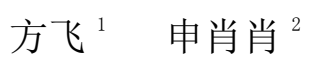 \\ 1) 山东工商学院外国语言文学系, 烟台, 山东, 中国 \\ 2) 烟台大学文经学院外国语言文学系, 烟台, 山东, 中国}

摘 要 随着全球经济一体化的加深, 各国之间商务、旅游、文化、政治等各方面交流日益频繁。作为外国游客在中国的临时住 所, 酒店及其所提供的双语服务和环境的重要性日益凸显。本文以文本类型学为理论基础, 探讨了酒店公示语的文本特点、文本功 能、翻译原则和目前所存在的问题, 旨在为目前酒店双语环境的营造提供参考性建议。

关键词 文本类型, 文本功能, 互文性, 翻译原则, 借译

1. 引言

公示语（public sign），又称“标志语”、“标识语”、 “标语”, 是指在公共场所展示的文字, 具有特殊的交际功 能, 以及提供信息和完成指令的作用 ${ }^{1}$ 。公示语经常出现在 地铁、机场、加油站、旅游景点、酒店、度假村等公共设 施和旅游胜地, 除了文字外, 还常常配有通用标志。近年 来, 随着全球化的发展, 我国经济和全球经济日趋一体化, 各种国际赛事、博览会、展览会的举办进一步加深了国内 外的经济、文化和体育交流, 公示语翻译的重要性日益凸 显出来。公示语翻译研究已经引起众多专家和学者们的关 注, 他们主要从语用学、文本类型学、目的论、交际翻译

1 罗选民黎土旺. “关于公示语翻译的几点思考”. 《中国翻译》. 2006 .
理论、翻译的互文性以及生态翻译学的视角对公示语的翻 译现状和标准进行了探讨研究。本文拟采用文本类型学理 论分析酒店公示语的文体特点、文本功能、翻译原则和存 在的问题, 以期对酒店公示语这一特殊的公示语文本翻译 进行初步的探讨, 提出可行的翻译方案。

\section{2. 酒店公示语的文本特点}

罗选民 (2006：67) 在首届全国公示语翻译研讨会上作 了《关于公示语翻译的几点思考》的主旨发言, 后发表于 《中国翻译》, 在文中他指出公示语的风格特点主要有简洁 性、规约性和互文性。吕和发 (2004：40) 指出, “英语公 示语具有严格的规范性、标准型、沿袭性, .....”。樊桂 芳（2010:47）指出 “公示语在语体上有简洁性、直接性和 互文性的特点。” 酒店公示语作为公示语的一种, 具有简 
洁性、规范性和互文性, 并且和其他旅游公示语相比, 这 些特点更为突出。因为, 景点公示语或公共设施公示语往 往比较能够体现一个国家的文化特色, 知识性介绍或者补 充显得尤为重要, 因此我们在景点常常能够看到大段的文 字介绍, 讲述景点背后的典故和来源。但酒店往往不涉及 这些文化信息, 其所包含的住宿、休闲、娱乐等功能在全 球所有酒店中具有高度的一致性, 因此在酒店公示语翻译 中要特别突出其简洁性、规范性和互文性。

\section{3. 酒店公示语的文本功能}

德国翻译功能学派代表人物 Katharina Reiss（卡塔 琳娜 - 赖斯）根据语言的三种功能: 信息功能、表情功能 和感染功能, 将文本分为信息类文本、表情类文本和操作 类文本。信息类文本主要目的是准确地传达文本所包含的 信息, 主要包括经济类、法律类、新闻类文本等, 如产品 说明书。表情类文本主要目的是表情达意, 让读者完全领 悟到文本中的思想感情、艺术美感等, 如小说。感染类文 本目的是让读者在阅读完成后采取文本后行动, 因此又称 操作类文本, 如广告。

赖斯认为现实中的一个文本很少体现单一的文本功 能, 往往是多种功能并存, 以某种功能为主。酒店公示语 和其他公示语一样兼具指示、提示、限制、强制和召唤功 能 $^{2}$ 。就文本所具有的三种功能而言, 酒店公示语不同于景 点公示语, 它的表情功能较弱, 而其主要的目的是指示酒 店布局、提示顾客采取或禁止某种行为, 因此以感染功能 为主, 兼有信息功能。

\section{4. 酒店公示语的翻译原则}

酒店公示语的翻译需要遵循以下 4 个原则: 1 、简洁性 原则; 2、规范性原则; 3、统一性原则; 4、互文性原则。 简洁性主要体现在公示语中多用名词、动词、动名词、短 语和缩略语, 如 Check-in, Check-out, Exit 等。另外, 酒店公示语常常是文字和通用标志共用, 直观地向顾客提 供指示功能。规范性是指公示语是一种独特的文体, 翻译 之后的公示语要符合目的语读者的语言使用习惯和文化心 理。统一性主要针对专有名词的翻译, 在同一文本中同一 个或同一类专有名词尽量采用同一个译名或同一种翻译方 法来翻译。

互文性体现在公示语的产生和解读都是某种文化中各 种文本相互作用的结果, 因此我们在翻译时应该把它放在 原语文化中进行互文性解读, 完成意义构建。在此基础上,

\footnotetext{
2 丁大刚. 《旅游英语的语言特点与翻译》上海交大出版社, 2008 .
}

利用目的语先期文本的互文性，找到目的语中与之相对应 的已有的固定用法重构文本。因此公示语翻译方法中首选 借译。而对于极具民族文化特色的公示语, 在目的语中往 往会出现零对应的情况, 一般可采用意译或拼音加注的方 式翻译。酒店公示语不仅与所在语言文化具有互文性, 即 使在不同文化间也具有较强的互文性, 因此翻译时, 一般 采用借译的方法。

\section{5. 酒店公示语翻译中存在的问题}

\section{1 信息的篡改与丢失}

酒店公示语的主要功能之一是准确地向顾客传达方 位、处所、服务等信息, 以方便顾客的住宿和休闲, 因此 如果翻译过程中丢失信息就会造成交际失误, 影响交际效 果和顾客对酒店的双语环境印象。例如, 在有的酒店 “廊 灯” 被译为 “Corridor”, “灯” 的意思完全丢失。而 “房 务部” 被译为 “Front Office Department”, 相信不少外 国顾客会无法理解什么是 “front office”, 因此不如改 译成 “Room Service Department”。

\section{2 语言的失当与错误}

有时由于译者对原文理解的偏差或者语言使用水平参 差不齐, 译文常常会出现语法、词汇甚至拼写错误, 这是 应该而且完全可以避免的。比如: 在走过庭院的桥面时, 我们常常可以看到这样的提示语: “水深地滑 注意安全”。 在一家酒店, 这句话被译成: “DEEP WATER/SMOOTH GROUND PLEASE BE CAREFUL”。虽然译者按照字字对 应的方式将原文译出, 但是这里却出现了翻译的错误。 “smooth” 是用来形容物体表面光滑、没有障碍, 触感较 好, 与原文中的 “地滑” 是不对应的。再如图 1, “小心地 滑” 的提示语被译为 “小心地去滑倒”, 让人啼笑皆非。

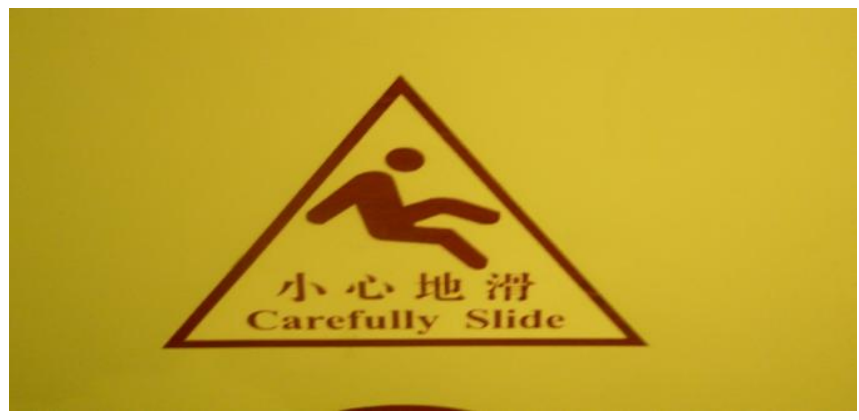

图 1 烟台中心大酒店公示语 


\section{3 忽视互文性借译}

在酒店的楼梯上我们常常可以看到 “请您小心台阶!” 这句公示语, 紧接着下面的译文是 “Please be careful of the step! ”从语法角度讲, 这种译法是完全可以的, 但 是译者并未从互文性角度考虑目的语中已有的固定译法, 比如上面的句子可以译成: Mind your step. 同样的还有, 在电梯旁有时我们可以看到 “员工专用”, 提示顾客电梯只 供职工使用, 有的酒店将其译为: Specialized for Staff. 也并未考虑借译: Staff only.

互文性借译充分考虑了目的语读者的语言习惯和惯用 法, 这样不仅体现出译文的地道, 也能充分展示公示语和 国际接轨的程度。

\section{4 译文缺乏统一性}

如图 2, 在同一家酒店，对于 “购物中心” 和 “商务 中心” 的翻译, 译文分别运用了英式英语拼写法 (Centre) 和美式英语拼写法 (Center), 这违反了译名统一的原则。 建议在同一场合下, 翻译公示语时应该保持译法和译名的 一致性。

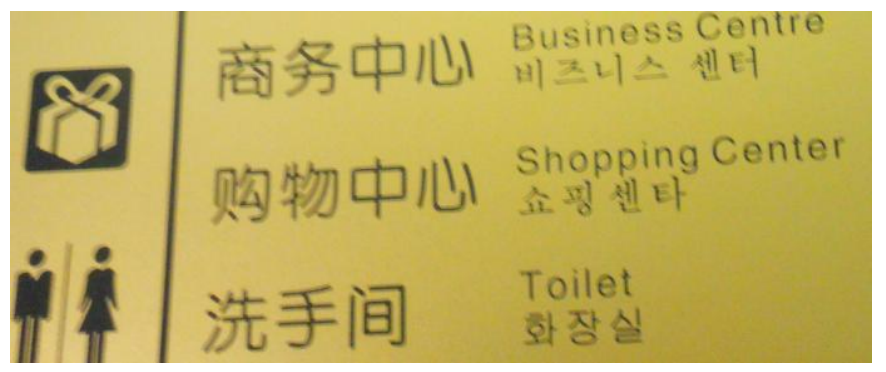

图 2 烟台碧海饭店公示语

\section{6. 结语}

本文以 Katharina Reiss 的文本类型学为视角分析了 酒店公示语的文体特点、文本功能、翻译原则和目前存在 的问题。酒店公示语具有简洁性、规范性和较强的互文性,
文本功能以感染功能为主, 兼具信息功能, 因此在翻译酒 店公示语时我们应遵循简洁性、规范性、统一性和互文性 的翻译原则, 充分考虑到酒店公示语在不同文化中的互文 性和相通之处, 以及目的语读者的语言使用习惯，首选借 译的翻译方法, 以期实现酒店公示语的规范化翻译, 避免 出现信息丢失、语言表述错误等常见错误, 为规范酒店双 语环境, 展现城市形象, 文化底蕴与国际化程度而尽绵薄 之力。

\section{参考文献(References)}

[1] Meifang Zhang. Investigating the Languages and Translations of Public Notices in Macao. Shanghai Journal of Translators, 2006 (1): 29-34.

[2] Yingchun Liu, Haiyan Wang. A Study of the Translation of Public Notices Based on Text Types. Chinese Translators Journal, 2012 (6): 89-92.

[3] Xuanmin Luo, Tuwang Li. Reflections on the Translation of Public Signs. Chinese Translators Journal, 2006 (4): 66-69.

[4] Dagang Ding. Linguistic Features \& Translation of Tourism Texts. Shanghai: Shanghai Jiao Tong University Press, 2008.

[5] Hefa Lv. C-E Translation of Public Signs. Chinese Science \& Technology Translators Journal, 2004 (1): 38-40.

[6] Guifang Fan. Translation of Public Signs from the Perspective of Intertextuality. Chinese Science \& Technology Translators Journal, 2010 (4): 47-50.

[7] Youxue Shao. Rethinking on the C-E Translation of Public Signs. Chinese Science \& Technology Translators Journal, 2009 (2): 48-51.

[8] Xueyun He. The Present Situation of C-E Public Signs Translation and the Communicative Translation Strategy. Foreign Languages and Their Teaching, 2006 (3): 57-59.

[9] Reiss, Katharina. Translation Criticism: The Potentials and Limitations. London and New York: Routledge, 2001. 